\title{
Exciton-Plasmon-Photon Conversion in silver nanowire: polarization dependence
}

\author{
Lu-Lu Wang, ${ }^{1}$ Xi-Feng Ren,, , 4 Ai-Ping Liu, ${ }^{1}$ Liu Lv, ${ }^{1}$ Yong-Jing Cai, ${ }^{1}$ Guangcan Guo, ${ }^{1}$ and Guoping Guo ${ }^{1}$ \\ ${ }^{1}$ Key Laboratory of Quantum Information, University of Science and Technology of China, Hefei 230026, People's Republic of China
}

\begin{abstract}
Polarization dependence of the exciton-plasmon-photon conversion in silver nanowire-quantum dots structure was investigated using a scanning confocal microscope system. We found that the fluorescence enhancement of the CdSe nanocrystals was correlated with the angle between the excitation light polarization and the silver nanowire direction. The polarization of the emission was also related with the nanowire direction. It was in majority in the direction parallel with nanowire.
\end{abstract}

PACS numbers: 78.66.Bz,73.20.MF, 71.36.+c

Surface plasmon polaritons (SPPs), collective oscillating electrons excited by electromagnetic field, have been studied for decades and a great of interest has been injected into this area. SPPs are involved in a wide range of phenomena [1, 2], including nanoscale optical waveguiding [3], perfect lensing [4], extraordinary optical transmission [5], subwavelength lithography [6], and ultrahigh sensitivity biosensing [7]. Analogous to optical fiber in nanoscale, plasmonic waveguide has received more and more attention since SPPs can propagates in nanoscale structures. Among various plasmonic waveguides, silver nanowires have some unique properties that make them particularly attractive, such as low propagating loss due to their smooth surface and scattering of plasmons to photons only at their sharp ends[8, 9]. Recently, not only the efficient coupling method was proposed [10-12], but also the correlation between incident and emission polarization in Ag nanowire was studied[13].

Interaction of surface plasmons and quantum dots (QDs) attracted more and more attention recently because of the importance of QDs in quantum information [14]. The metallic nanostructures can be used to modify the emission properties of QDs. The enhancement or quenching of QDs emission in various structures has been researched in many works 15 , 1720]. Recently, the interaction of propagating SPs and QDs has also been investigated. Akimov et al. developed a single optical plasmons by coupling the SPPs of silver nanowire with a single quantum dot[21]. Wei et al. showed the loss of energy and change of spectrum when emission of QDs propagated along the silver nanowires[22].

Polarization is an important property of photons, thus the polarization properties of the exciton-plasmon-photon conversion of QDs in the metallic nanowire should be studied intensively. In this paper, we studied the polarization dependence of the efficiency of exciton-plasmon-photon conversion in silver nanowire-quantum dots structure. The results showed that the enhancement factor of QDs fluorescence was correlated with the angle between the polarization of the laser and the direction of the nanowire. Due to the antenna effect of the nanowire, the polarization of the fluorescence was also changed with the direction of the nanowire.

The $15 \mathrm{~nm}$ silica capped Ag nanowires (about $300 \mathrm{~nm}$ in diameter and $10 \mu \mathrm{m}$ in length) were prepared by using a wet chemistry method described by Sun and Xia[23, 24]. This
nanowire-QDs distance was selected to get a high enhancement of fluorescence of QDs[15, 21]. A drop of solution of these $\mathrm{Ag}$ nanowires in ethanol was deposited on a clean glass substrate and dried naturally. Then a drop of CdSe/ZnS QDs (the fluorescence emission wavelength centered at $610 \mathrm{~nm}$ ) was deposited above the Ag nanowires and covered with another glass slide immediately. The coverslip should be lightly pressed in order to get uniform distribution of the QDs. After the sample was dry, the capped glass slide was abandoned while the silver nanowires covered with QDs on the substrate were used for the following measurement.

The experimental setup was shown in Fig. 1. The sample placed on a three-dimensional piezoelectric transition (PZT) stage were excited by a continuous-wave laser at $532 \mathrm{~nm}$, whose polarization can be adjusted by a $\lambda / 2$ plate (work wavelength $532 \mathrm{~nm}$ ) behind a polarization beam splitter(PBS). The laser light was focused on the sample by a $60 \times$ oil objective $(\mathrm{NA}=1.35)$, and the fluorescence from the QDs were collected with the same objective and detected by a single photon detector(SPD, SPCM-15, PerkinElmer Optoelectronics, Canada). A green-orange dichroic plate was used to reflect the laser light and transmit the fluorescence from QDs(the emitting peak is centered at $610 \mathrm{~nm}$ at room temperature). Spatial filtering was realized by the confocal lens with a pinhole(diameter $30 \mu \mathrm{m}$ ) in center. This setup can collect the light from an area about $1.5 \mu \mathrm{m}$ in diameter on sample surface. The area can be selected artificially by moving the the front lens of the confocal lens system. A narrow band filter(center wave-

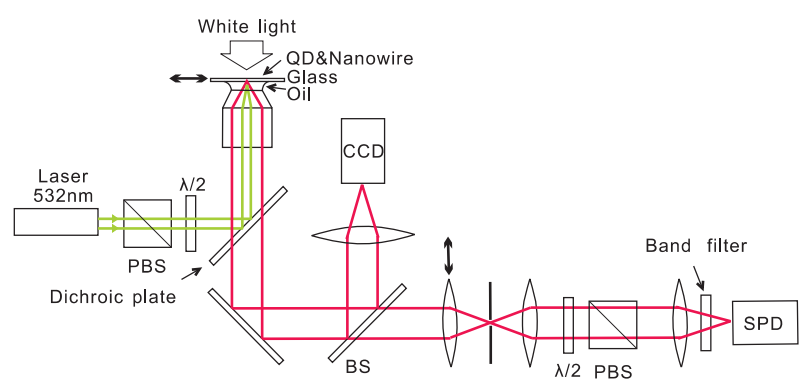

FIG. 1: (Color online) Sketch of the experimental setup. A homemade scanning confocal microscope was used to investigate the polarization dependence of exciton-plasmon-photon conversion in silver nanowire-QDs structure. 
length $633 \mathrm{~nm}$ ) was also used to reflect the illuminated light before the SPD. The polarization of the fluorescence was analyzed by another $\lambda / 2$ plate (work wavelength $=633 \mathrm{~nm}$ ) combined with a PBS. A CCD camera was used for direct imaging.

Fig. 2(a) gave the CCD picture of a $\mathrm{Ag}$ nanowire with length about $14 \mu \mathrm{m}$. When the $532 \mathrm{~nm}$ laser was focused on the middle of the nanowire, the ends of nanowire kept dark(Fig. 2(b)), which means that surface plasmons cannot be excited in the nanowire. The reason was that the momentum difference between the surface plasmons and photons was not bridged due to the smooth surface of nanowire. This phenomenon was observed in many works [8, 9]. However, the observed phenomenon changed as the nanowire was coated by the QDs. When using a $610 \pm 17 \mathrm{~nm}$ band-pass filter in front of the CCD to block the laser light, we can see the fluorescence on the ends of nanowire obviously(Fig. 2(c)). Contrast with Fig. 2(b), we considered that the fluorescence light at the ends of nanowire were came from the propagating SPPs excited by QDs' fluorescence around the nanowire but not the $532 \mathrm{~nm}$ laser. We also scanned the sample in the area of $30 \times 10 \mu \mathrm{m}$ (the scan step is $0.2 \mu \mathrm{m}$ ) around the $\mathrm{Ag}$ nanowire and collected the fluorescence. The result was shown in Fig. 2(d). Strong fluorescence near the nanowire can be clearly observed. The bright spot outside the nanowire in Fig. 2(d) was attributed to the enhancement of nanoparticle doping.

The relation between the intensity of the fluorescence and the cross angle of silver nanowire and laser light polarization was investigated by rotating the first $\lambda / 2$ plate $(532 \mathrm{~nm})$. By moving the front lens of the confocal system, we can selectively collect the fluorescence from the middle part(excitation spot) or one end of the nanowire. For both cases, different counts were recorded by the SPD for various polarization of the excitation light, as shown in Fig. 3. $\theta$ was defined as the

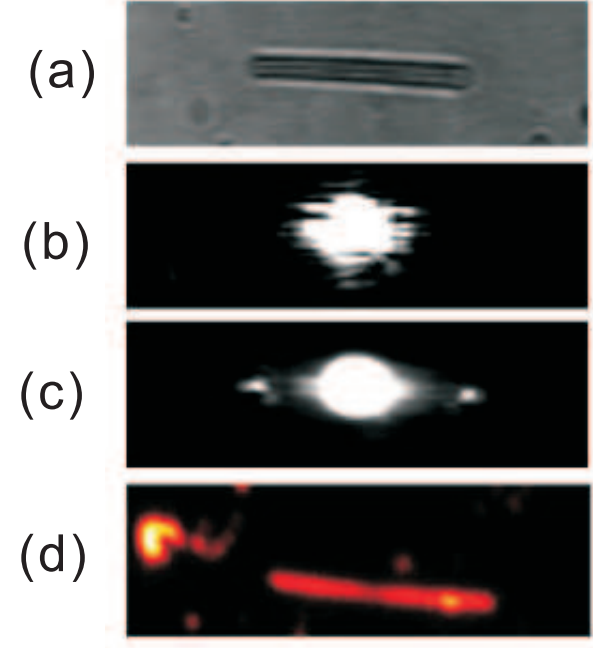

FIG. 2: (Color online) (a) CCD image of a silver nanowire.(b) Image with $532 \mathrm{~nm}$ laser focused on the middle of the nanowire. (c) Image of the QDs emission as the same setup as case b. (d) Scanning image of the QDs emission. cross angle between the silver nanowire and the polarization of excitation laser. The normalized counts from the middle and right end of the Ag nanowire were nearly coincident, both of them came to maximum when the polarization of laser was parallel with the nanowire, and to minimum when they were perpendicular. They were nicely fitted with cosine function of $\theta$. $I_{\max } / I_{\text {low }}$ were about 1.34 for the both cases of the middle part and the right end, where $I_{\max }$ and $I_{l o w}$ were the maximum and minimum counts respectively. The same values supported that the fluorescence from the end of the nanowire came from propagating SPs excited by the QDs near the Ag nanowire. This polarization dependence may be due to the different excitation efficiency of the SPPs, which contributed to the enhancement of the fluorescence [15, 16.

The polarization of the fluorescence from the middle of the nanowire (excitation spot) and one end of the nanowire were also analyzed. By rotating another $\lambda / 2$ plate $(633 \mathrm{~nm})$ before the SPD, we can selectively detected the fluorescence in different polarization. Three cases for the excitation laser polarization with $\theta=0^{\circ}, 45^{\circ}$ and $90^{\circ}$ were discussed as shown in FIG. 4. $\phi$ was defined as the angle between Ag nanowire and polarization of detected fluorescence. In all the cases, the fluorescence showed maximum or minimum counts as the detected polarization was parallel or perpendicular with the nanowire respectively. It means that the fluorescence was mainly polarized towards the direction of nanowire. This phenomenon may came from the antenna effect of the metal nanowire structure, which was similar with the result of $\lambda / 2$ dipole antennas in ref. [25]. The polarization ratio $I_{\|}^{\text {mid }} / I_{\perp}^{\text {mid }}$ is $1.39\left(\theta=0^{\circ}\right), 1.65\left(\theta=45^{\circ}\right), 1.74\left(\theta=90^{\circ}\right)$ respectively, where the $I_{\|}\left(I_{\perp}\right)$ was the intensity of fluorescence with the polarization parallel(perpendicular) with the nanowire. The similar result was found for the fluorescence from the right end of the nanowire, as shown in Fig. 5, while the polarization ratio $I_{\|}^{\text {end }} / I_{\perp}^{\text {end }}$ was changed to $1.76\left(\theta=0^{\circ}\right), 2.01\left(\theta=45^{\circ}\right), 2.13$ $\left(\theta=90^{\circ}\right)$. They were all higher than the previous case, which

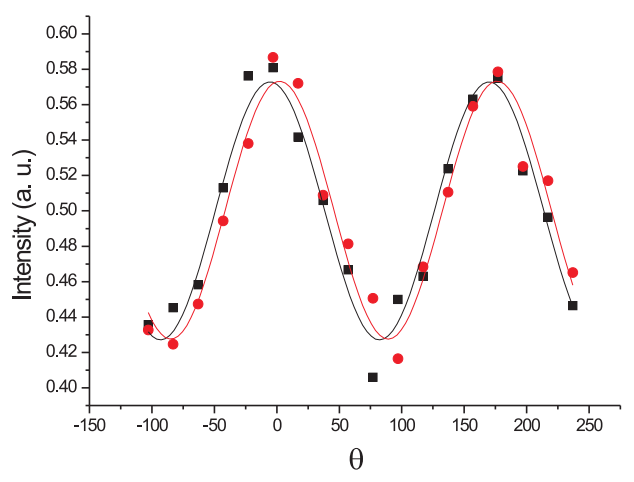

FIG. 3: (Color online) Intensity of fluorescence from the middle(black square dots) and the right end of the nanowire(red round dots) as the function of $\theta$. The results were nicely fitted with cosine function. 
may be attributed to the polarization selection in the propagation of SPPs[13].

Several nanowires were investigated using the same method and shown the similar results. The only differences were the unequal polarization ratio $I_{\text {max }} / I_{\text {low }}$ and $I_{\|} / I_{\perp}$, which were related to the length and diameter of nanowires. As a comparison, we also studied the cases of pure QDs sample using the same setup. It was found that not only the intensity of the fluorescence was independent of the polarization of the excitation light, but also the polarization of the fluorescence was random. This was quite different with the silver nanowire-quantum dots system, consequently, the observed results shown in the Figs. 3-5 were caused by the presence of the SPPs of the nanowire.

In summary, we investigated the polarization dependence in exciton-plasmon-photon conversion in silver nanowirequantum dots system. Due to the effect of the SPPs, the whole process was quite different from the excitation of the pure QDs. Besides the strong correlation between the fluorescence enhancement factor and the polarization of the excitation laser, the polarization of the fluorescence itself was also related with the direction of the nanowire. Our results will be useful in the further research on the interactions of SPs and QDs.

The authors thank Chang-Ling Zou and Jin-Ming Cui for useful discussion. This work was funded by the National Basic Research Programme of China (Grants No.2011CBA00200), the Innovation funds from Chinese Academy of Sciences, and the National Natural Science Foundation of China (Grants No.10904137), Anhui Provincial Natural Science Foundation(Grants No. 090412053) and Science and Technological Fund of Anhui Province for Outstanding Youth(Grants No.2009SQRZ001ZD).

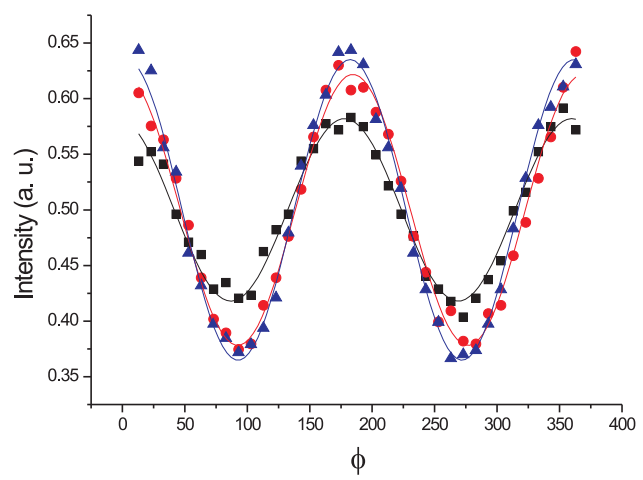

FIG. 4: (Color online) Intensity of fluorescence from the middle of the nanowire as the function of $\phi$. For black square dots, red round dots and blue triangle dots, $\theta$ were 0,45 and 90 degree respectively.
* Electronic address: renxf@ ustc.edu.cn.

[1] W. L. Barnes, A. Dereux, and T. W. ebbesen, Nature. 424, 824 (2003).

[2] E. Ozbay, Science. 311, 189 (2006).

[3] S. I. Bozhevolnyi, V. S. Volkov, E. Devaux, J. Y. Laluet, and T. W. Ebbesen, Nature. 440, 508 (2006).

[4] J. B. Pendry, Phys. Rev. Lett. 85, 3966 (2000).

[5] T. W. Ebbesen, H. J. Lezec, H. F. Ghaemi, T. Thio and P. A. Wolff, Nature. 391, 667 (1998).

[6] N. Fang, H. Lee, C. Sun, and X. Zhang, Science. 308, 534 (2005).

[7] B. Liedberg, C. Nylander, and I. Lundstrom, Sensors and Actuators. 4, 299 (1983).

[8] A. W. Sanders, D. A. Routenberg, B. J. Wiley, Y. N. Xia, E. R. Dufresne, and M. A. Reed, Nano Lett. 6, 1822 (2006).

[9] H. Ditlbacher, A. Hohenau1, D. Wagner, U. Kreibig, M. Rogers, F. Hofer, F. R. Aussenegg, and J. R. Krenn, Phys. Rev. Lett. 95, 257403 (2005).

[10] M. W. Knight, N. K. Grady, R. Bardhan, F. Hao, P. Nordlander, and N. J. Halas, Nano Lett. 7, 2346 (2007).

[11] A. L. Pyayt, B. J. Wiley, Y. N. Xia, A. T. Chen, and L. Dalton, Nature nanotechnology 3, 660 (2008).

[12] C.H. Dong, X. F. Ren, R. Yang, J. Y. Duan, J. G. Guan, G. C. Guo, and G. P. Guo Appl.Phys.Lett. 95, 221109(2009).

[13] Z. P. Li, K. Bao, Y. R. Fang,Y. Z. Huang, P. Nordlander, and H. X. Xu, Nano Lett. 10, 1831 (2010).

[14] R. Hanson, L. P. Kouwenhoven, J. R. Petta, S. Tarucha, and L. M. K. Vandersypen, ReV. Mod. Phys. 79, 1217 (2007).

[15] Y. Fedutik, V. V. Temnov, O. Schops, and U. Woggon, Phys. Rev. Lett. 99, 136802 (2007).

[16] C. L. Zou, F. W. Sun, Y. F. Xiao, C. H. Dong, X. D. Chen, J. M. Cui, Q. H. Gong, Z. F. Han, and G. C. Guo, Applied Physics Letter, 97, 183102(2010)

[17] K. Matsuda, Y. Ito, and Y. Kanemitsu, Appl. Phys. Lett. 92, 211911 (2008).

[18] A. Ueda, T. Tayagaki, and Y. Kanemitsu, Appl. Phys. Lett. 92, 133118 (2008).

[19] A. O. Govorov, G. W. Bryant, W. Zhang, T. Skeini, J. Lee, N. A. Kotov, J. M. Slocik, and R. R. Naik, Nano Lett. 6, 984 (2006).

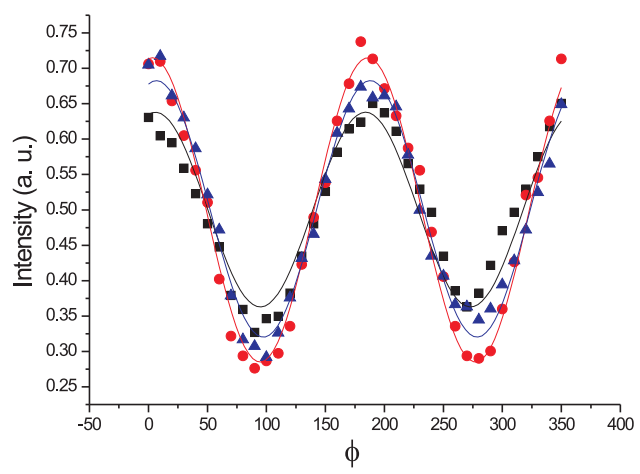

FIG. 5: (Color online) Intensity of fluorescence from the right end of the nanowire as the function of $\phi$. For black square dots, red round dots and blue triangle dots, $\theta$ were 0,45 and 90 degree respectively. 
[20] X. W. Wu, M. Gong, C. H. Dong, J. M. Cui, Y. Yang, F. W. Sun, G. C. Guo, and Z. F. Han, Opti. Express 18, 6340 (2010).

[21] A. V. Akimov, A. Mukherjee, D. E. Chang, A. S. Zibrov, P. R. Hemmer, H. Park, and M. D. Lukin, Nature. 450, 402 (2007).

[22] H. Wei, D. ratchford, X. Q. Li, H. X. Xu, and C. K. Shih, Nano Lett. 9, 4168 (2009).
[23] Y. G. Sun, and Y. N. Xia, Adv. Mater. 14, 833 (2002).

[24] Y. D. Yin, Y. Lu, Y. G. Sun, and Y. N. Xia, Nano Lett. 2, 427 (2002).

[25] A. G. Curto, G. Volpe, T. h. Taminiau, M. P. Kreuzer, R. Quidant, and N. F. van Hulst, Science. 329, 930 (2010). 